\title{
First clinical results with a real time, electronic imager as an aid to colonoscopy
}

\author{
B P Saunders, G D Bell, C B Williams, J S Bladen, A P Anderson
}

\begin{abstract}
The early clinical results are described of a real time, electromagnetic imaging system as an aid to colonoscopy. After gaining experience with the use of the system, one experienced endoscopist was randomised to perform consecutive colonoscopies either with $(n=29)$ or without $(n=26)$ the imager view. All procedures were recorded on computer disk and replayed for retrospective analysis. Total colonoscopy was achieved in all patients except one (imager view not available). Comparing intubation time and duration of loop formation per patient, there was no significant difference between the two study groups. The number of attempts taken to straighten the colonoscope per patient, however, was less when the endoscopist was able to see the imager view, $\mathbf{p}=0.03$. Hand pressure was also more effective when the endoscopist and endoscopy assistant could see the imager display, $p=0 \cdot 02$. Preliminary experience suggests that real time, electronic imaging of colonoscopy is safe, effective, and will improve the accuracy of the procedure.

(Gut 1995; 36: 913-917)
\end{abstract}

Keywords: colonoscopy, real time electronic imager.

Correspondence to: Dr B P Saunders, St Mark's Hospital, City Road, London EC1V 2PS.

Accepted for publication 24 October 1994
It is now widely recognised that colonoscopy is the investigation of choice for most diseases of

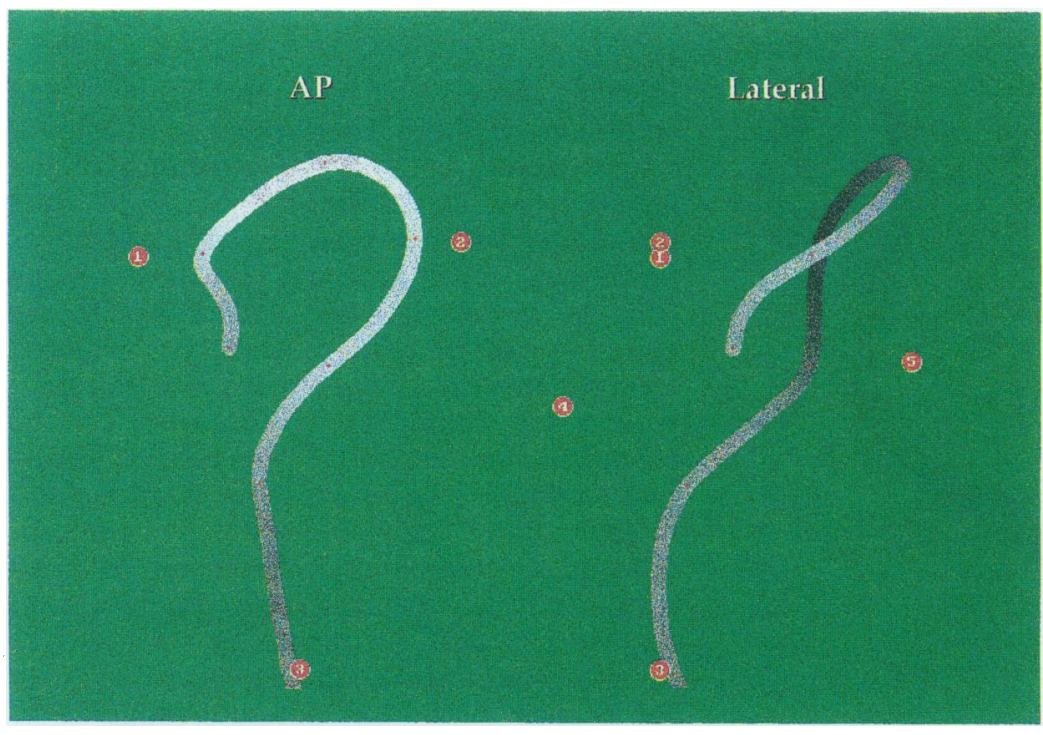

Figure 1: Imager display of caecal intubation (AP and lateral views) showing position of body markers $(1=10$ th rib in the right mid-axillary line, $2=10$ th rib in the left mid-axillary line, 3=anus, 4=3rd lumbar spine, 5=umbilicus). The colonoscope shaft is represented by differential grey shading, ranging from white (nearest to the viewer) to black (farthest from the viewer). the colon. ${ }^{1-4}$ It remains a technically demanding procedure, however, which is both difficult to learn and time consuming to teach. ${ }^{5}$ Most of the difficulties met during colonoscopy are caused by recurrent looping of the colonoscope in the surprisingly variable colonic anatomy encountered. ${ }^{6}$ In most cases, once a loop has formed it must be straightened before the tip of the instrument can be advanced and total colonoscopy achieved. The manoeuvres that successfully straighten the colonoscope are dependent upon the type of loop that has formed. ${ }^{7}$ Therefore it is desirable to be able to visualise the configuration of the colonoscope inside the patient as well as to see the anatomical location of the colonoscope tip. We have previously described a new, non- $x$ ray method for imaging endoscopes. ${ }^{89}$ In this paper we report on our up dated clinical experience with a real time version of the former system as an aid to colonoscopy.

\section{Method}

Real time electromagnetic imaging: principle of the method

Three sets of generator coils situated beneath the endoscopy table, sequentially produces pulsed, low strength (about $1 \times 10^{-6}$ that of a standard magnetic resonance scan) electromagnetic fields external to the patient. (Bladen JS, et al. Conference record of the 1993 IEEE Nuclear Science Symposium and Medical Imaging conference). These fields are detected by a series of 15 sensor coils $(1 \mathrm{~cm}$ in length by $2 \mathrm{~mm}$ wide), positioned at $12 \mathrm{~cm}$ intervals along the length of a catheter that is inserted down the biopsy channel of the colonoscope. From the electrical signal produced in the sensor coils the exact position and orientation of each sensor can be calculated. By fitting a smooth curve through each of the calculated points a computer graphics image of the colonoscope shaft is built up and displayed on a computer monitor. A three dimensional effect is created by differential grey shading (Fig 1) and the image is updated every 0.2 seconds, so that the system is effectively real time. Images obtained can be stored on computer disk (about six entire patient records can be stored on one standard floppy disk) and replayed for teaching or research purposes in a Windows (Microsoft Corporation) based viewing program. The viewing program allows measurements to be taken between points of interest and snap shot images to be imported into other Windows based graphics programs. 
TABLE I Patient details

\begin{tabular}{lll}
\hline & $\begin{array}{l}\text { Imager } \\
(n=29)\end{array}$ & $\begin{array}{l}\text { No imager } \\
(n=26)\end{array}$ \\
\hline Sex & & \\
$\quad$ Male & 14 & 13 \\
$\quad$ Female & 15 & 13 \\
Age & & \\
$\quad$ Mean (y) & 61 & 59 \\
$\quad$ Range (y) & $37-89$ & $34-83$ \\
Post surgical history & 2 & 0 \\
$\quad$ Hysterectomy & 3 & 2 \\
Right hemicolectomy & 4 & 1 \\
Anterior resection & 3 & 4 \\
Other abdominal surgery & & \\
\hline
\end{tabular}

Preliminary clinical evaluation

The electronic imager was evaluated by one experienced (>1000 colonoscopies performed) endoscopist over a six week period at Ipswich General Hospital. All patients gave informed consent and the study was approved by the local ethics committee. In 61 consecutive colonoscopies the endoscopist and endoscopy personnel were randomised to perform the procedure either with or blindly without the imager view. Three patients were excluded from the study because of failed preparation, two because of fixed impassable sigmoid colons secondary to diverticular adhesions and one because she subsequently refused the procedure. Relevant patient details were recorded (Table I). Pentax EC $3801 \mathrm{~L}$ video and FC 382FH fibreoptic colonoscopes were used for the examinations. Patients were placed in the left lateral position, intravenous access secured, and continuous nasal oxygen $(21 / \mathrm{min})$ given by nasal cannula. All patients received intravenous sedation by slow bolus injection and were monitored throughout the procedure with pulse oximetry. To provide more anatomical information on the imager display, at the beginning of each procedure a single sensor coil was used to register anatomic landmarks (marker 1-10th rib in the right mid axillary line, marker 2-10th rib in the left mid axillary line, marker 3 - anus, marker 4 - third lumbar vertebra, and

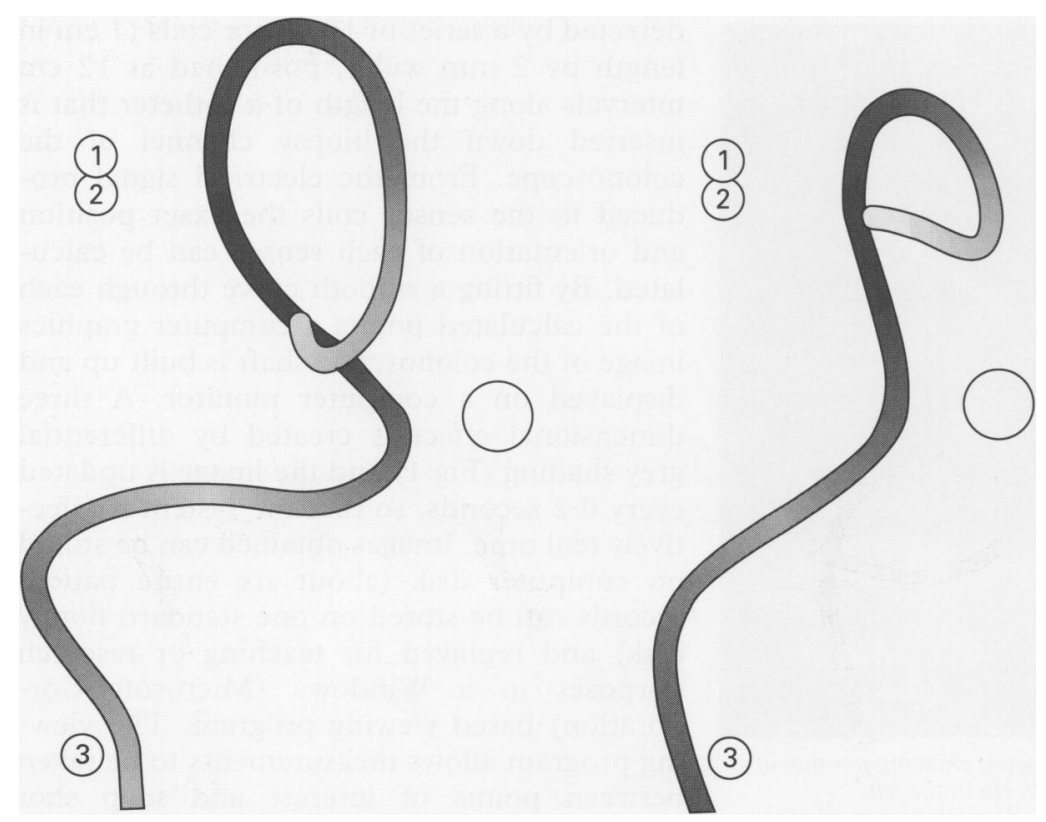

Figure 2: Accurate placement of the hand seen in the lateral view during abdominal pressure application. marker 5 - umbilicus), see Fig 1 . If the position of the patient changed during the procedure the same anatomic points were re-registered in the new patient position. The imager view was recorded continuously throughout intubation in both study groups, except when the catheter was withdrawn from the biopsy channel to facilitate suction, biopsy or polypectomy. For examinations in which the endoscopist was randomised to perform the procedure blindly, without the imager view he was asked at regular intervals by an independent observer, aware of the imager view, to give an estimation of the anatomical location of the colonoscope tip, whether he considered a loop had formed, and if so which type of loop he believed it to be. Changes in patient position were recorded. When abdominal pressure was requested, the endoscopy assistant placed a single sensor coil on the palm of the hand, (represented by a white sphere on the imager display) so that the position of the hand in relation to the colonoscope could be seen (Fig 2). After the examination the quality of bowel preparation, clinical findings, total time, and extent of the intubation were recorded. All images obtained were saved on computer disk and retrospectively analysed. For each procedure the type of loops that formed during intubation and their duration were recorded along with the number of attempts taken to successfully straighten the colonoscope and advance the colonoscope tip. In addition the accuracy of abdominal pressure and whether or not it was of benefit in advancing the colonoscope was recorded.

\section{Statistical analysis}

Unpaired $t$ tests were used to compare intubation time and duration of loop formation per patient between the two study groups. Categorical data were compared by Fisher's exact test after combining the data into 2 by 2 contingency tables. A p value was considered significant when $<0.05$.

\section{Results}

In 26 procedures the endoscopist and endoscopy assistant performed the colonoscopy blindly, without the assistance of the imager view. In 29 procedures both were allowed to see the imager view. Table II shows the findings at colonoscopy. Total colonoscopy (that is, insertion of the instrument to the caecum or surgical anastomosis) was achieved in all patients except one; in this case the endoscopist was without the imager view and the procedure was abandoned because of recurrent looping of the colonoscope in a long and mobile sigmoid colon. There was no significant difference in the time taken to complete intubation between the two study groups (mean intubation time without the imager $=12$ minutes, range 2-30 minutes, mean time with the imager view $=13$ minutes, range 4-30 minutes. The type and frequency of loops formed in the two study groups during intubation is shown in Table III and examples are shown in Figs 3 and 4. An ' $N$ ' or spiral loop 
TABLE II Findings at colonoscopy

\begin{tabular}{lcc}
\hline & $\begin{array}{c}\text { Imager } \\
(n=29)\end{array}$ & $\begin{array}{l}\text { No imager } \\
(n=26)\end{array}$ \\
\hline $\begin{array}{l}\text { Sedation } \\
\quad \text { Pethidine - mean (mg) }\end{array}$ & 47 & 48 \\
$\quad$ Midazolam - mean (mg) & $2 \cdot 9$ & $3 \cdot 1$ \\
Preparation & & \\
$\quad$ Acceptable & 20 & 16 \\
$\quad$ Poor & 9 & 10 \\
Diagnosis & 13 & 11 \\
$\quad$ Normal & 9 & 9 \\
Polyp & 5 & 2 \\
Cancer & 1 & 3 \\
$\quad$ Colitis & 0 & 1 \\
Angiodysplasia & & \\
\hline
\end{tabular}

occurred in the sigmoid colon in $65 \%$ of all patients and was the most common loop to occur at any time during intubation in both study groups. More patients examined without the imager view developed an alpha loop (35\%) compared with those examined with the imager (7\%), though this did not reach statistical significance $(p=0.08$ by Fisher's exact test). In three of four procedures where no loops formed at any stage during intubation the endoscopist had the benefit of the imager view. There was no difference between the two study groups with regard to the duration of time that the colonoscope was looping (mean time per patient without the imager view $=7 \cdot 3$ minutes, range $0 \cdot 3-28$ minutes, mean time per patient with the imager view $=6.2$ minutes, range 0.8-21 minutes). There were significantly fewer attempts at straightening the colonoscope, however, once a loop had formed when the imager view was available to the endoscopy personnel $(p=0.03$ by Fisher's exact test), Table IV.

Abdominal pressure was used on 10 occasions in both study groups (Fig 2). Advancement of the colonoscope tip and straightening of a loop associated with hand pressure occurred more frequently, however, when the endoscopy personnel could see the imager view, $p=0.02$ by Fisher's exact test (Table V).

Without the imager view, $42 \%$ of all loops that formed were incorrectly diagnosed by the endoscopist. A sigmoid spiral loop was correctly diagnosed on 24 of 28 occasions and
TABLE III Frequency of the different loops that occurred during intubation

\begin{tabular}{lcc}
\hline & $\begin{array}{c}\text { Imager } \\
(n=29)\end{array}$ & $\begin{array}{l}\text { No imager } \\
(n=26)\end{array}$ \\
\hline 'N' or sigmoid spiral & 22 & 28 \\
Alpha & 2 & 9 \\
Deep transverse & 7 & 5 \\
Reverse sigmoid spiral & 1 & 3 \\
Gamma & 1 & 1 \\
Reverse splenic flexure & 2 & 0 \\
Reverse alpha & 1 & 0 \\
Reverse transverse & 1 & 0 \\
Transverse spiral & 1 & 0 \\
Total & 38 & 46 \\
\hline
\end{tabular}

a deep transverse loop three times of five. Alpha looping occurred nine times but was only correctly recognised once while all three reverse sigmoid spiral loops and one gamma loop were incorrectly diagnosed. The endoscopist was incorrect in his estimation of the anatomical location of the colonoscope tip $13 \%$ of the time. In one patient a carcinoma at the sigmoid/descending junction was incorrectly located to the hepatic flexure.

\section{Discussion}

Over the past 20 years colonoscopy has superseded double contrast barium enema as the investigation of choice of the diseased colon. Demand for colonoscopy continues to grow. ${ }^{10}$ It remains a technically demanding procedure, however, despite improvements in colonoscope design and the introduction of video technology. The relative difficulty of the procedure is reflected by the published figures for total colonoscopy, which vary widely from 55-98\%. ${ }^{1-4} 11-14$ Even expert colonoscopists experience difficulty in the $10-20 \%$ of patients who possess a long or mobile colon. In nearly all patients during insertion, the colonoscope tends to loop, so that further pushing merely exerts force through the apex of the loop rather than to the tip of the instrument. To complete the examination such loops must be removed by variable combinations of clockwise/anticlockwise twist, withdrawal, changes of patient position, and application of abdominal pressure. Until now there has been no effective means of imaging the colonoscope inside

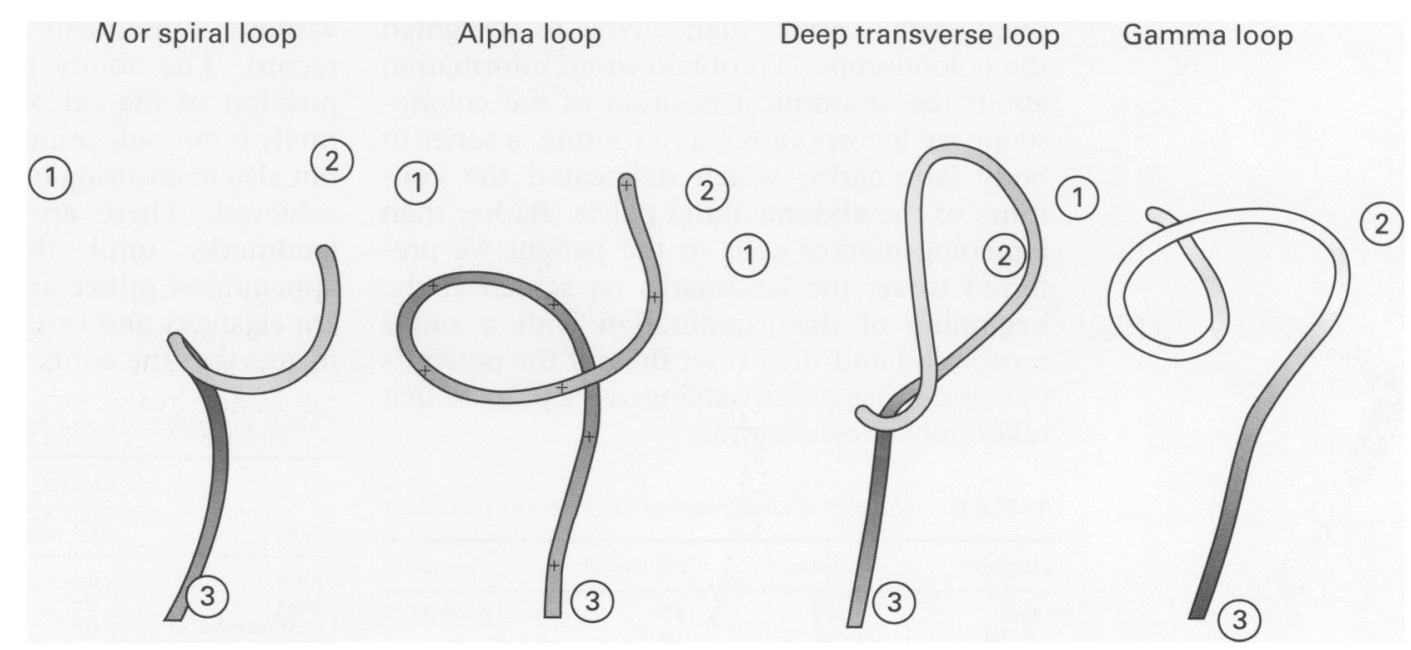

Figure 3: Examples of the most common loops seen during intubation. 


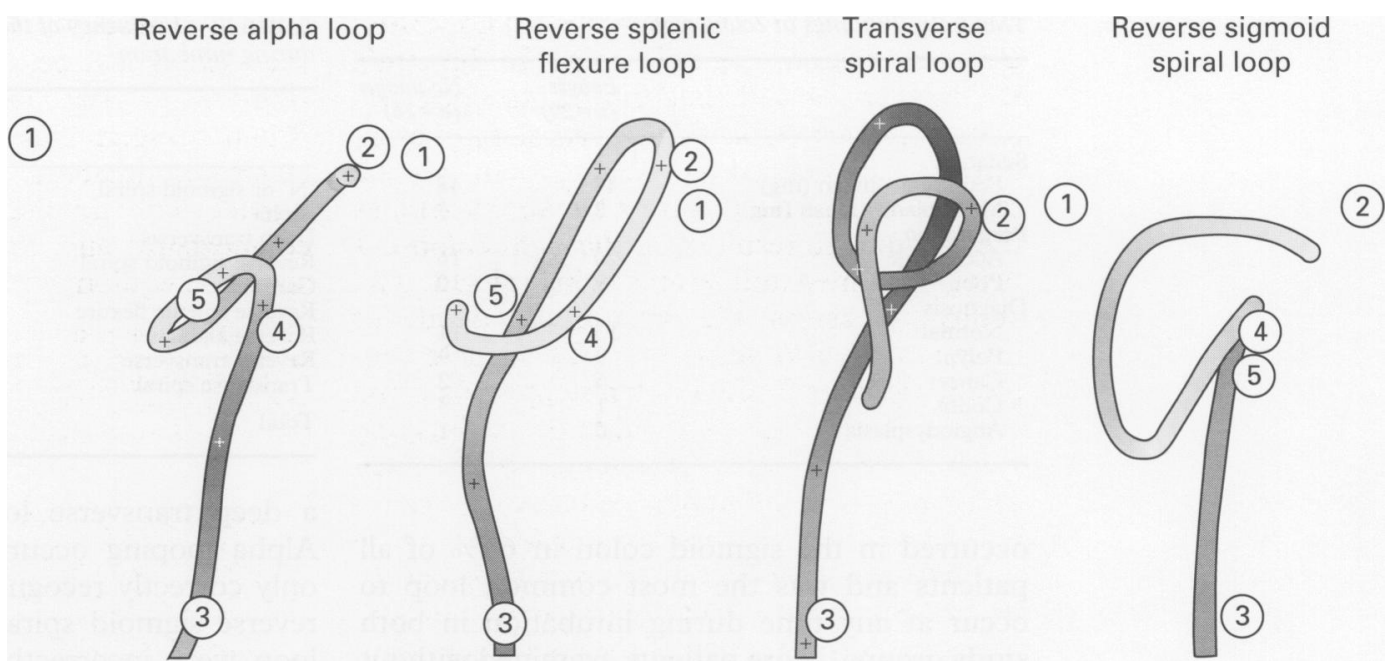

Figure 4: Examples of unusual loops seen during intubation.

the patient and therefore no accurate way of assessing loops. Fluoroscopy is available in a few limited centres but has important disadvantages in that the equipment is expensive and cumbersome, only provides fleeting, two dimensional views of the colonoscope, and its use presents a radiation risk to both patients and endoscopy staff. The removal of loops therefore has been undertaken blindly on a trial and error basis, expert colonoscopists being quicker and causing less pain than inexpert endoscopists or trainees by recognising loop formation earlier and finding the correct combination of straightening manoeuvres more rapidly.

Electronic imaging for the first time gives the endoscopist the ability to see the three dimensional configuration of the whole instrument and the exact location of its tip within the abdomen. The imager has now been used successfully in over 100 procedures and proves to be both robust and safe. The colonoscope configuration can be viewed from any position, but we found that for ease of recognition anteroposterior (AP) and lateral views were preferred. The lateral presentation in particular gave increased information about the depth and angulation of the colonoscope: often what appeared to be a shallow bend in the AP view was more acute when viewed laterally. The lateral view often permitted more accurate assessment of the correct manoeuvres to straighten the colonoscope. To obtain more information about the anatomical position of the colonoscope we incorporated, as a routine, a series of body landmarks, which delineated the contours of the abdomen and pelvis. Rather than attaching marker coils to the patient we preferred to set the landmarks on screen at the beginning of the examination with a single sensor coil and then reset them if the patient's position subsequently changed - a process that takes only a few seconds.

TABLE IV Number of straightening attempts per procedure

\begin{tabular}{llll}
\hline Attempts & Imager & No imager & Probability \\
\hline $0-5$ & 25 & 15 & $\mathrm{p}=0.03$ \\
$6-10$ & 4 & 10 & \\
$11-15$ & 0 & 1 & \\
\hline
\end{tabular}

Analysis of the two patient groups in this small pilot study, with an expert colonoscopist performing the procedures, has not shown that the imaging device significantly decreases the time taken to achieve total colonoscopy or that it reduces the duration of looping per patient. We have found, however, that with the benefit of the electronic imager, the number of attempts required to straighten the shaft is less and that assistant hand pressure is more effective in controlling loops. The imager permits comprehensive assessment of each loop that forms and so makes the manoeuvres to straighten the colonoscope logical and intuitive, rather than empirical.

By placing an additional sensor on the endoscopy assistant's hand, so that the position of the hand can be seen on the imager screen in relation to the colonoscope, abdominal pressure can be applied accurately over the apex of the loop to help to reduce it. It is sometimes obvious using the imager that the apex of the loop is deep in the abdomen so that abdominal pressure will be of little use and may even impede progress.

In this study the endoscopist was able to locate the tip of the colonoscope accurately $87 \%$ of the time without the benefit of the imager. He was incorrect about the anatomical location of one carcinoma, however, which would have resulted in confusion at surgery without subsequent analysis of the imager record. The ability to assess the anatomical position of the tip of the colonoscope accurately is not only important in localising lesions but also in ensuring total colonoscopy has been achieved. There are no reliable endoscopic landmarks until the ileocaecal valve or appendiceal orifice are reached and the inherent elasticity and variable mobility of the colon means that the entire $160 \mathrm{~cm}$ colonoscope can

TABLE V Effect of hand pressure (HP) during colonoscopy

\begin{tabular}{lcllll}
\hline & \multicolumn{2}{l}{ Imager } & & \multicolumn{2}{l}{ No imager } \\
\cline { 2 - 3 } \cline { 5 - 6 } \cline { 5 - 6 } Loop & HP used & Benefit & & HP used & Benefit \\
\cline { 1 - 2 } \cline { 5 - 6 } Spiral & 4 & 3 & & 6 & 1 \\
Alpha & 0 & 0 & & 3 & 0 \\
Deep transverse & 6 & 4 & & 0 \\
Total & 10 & $7(70 \%)$ & 10 & $1(10 \%)$ \\
\hline
\end{tabular}


be inserted with the colonoscope tip no further advanced than the sigmoid colon. In this study the imager showed that the endoscopist was always correct in assessing caecal intubation but less experienced endoscopists can mistake the mid-transverse colon or hepatic flexure as the caecum, so resulting in the right colon not being examined and lesions being missed. The ability to assess the anatomical depth of insertion is also important for flexible sigmoidoscopy or limited colonoscopy when the caecum is not reached. In addition the imaging system allows biopsy specimens to be taken accurately from different segments of the colon for surveillance and research purposes.

There are some limitations of the system. In its present form use of the electronic imager necessitates obstruction of the instrumentation channel by the sensor catheter, so impeding or sometimes preventing aspiration of air or fluid unless a two channel instrument is available. We anticipate that the chain of sensors will eventually be built into the shaft or inserted during repair or maintenance. This will have the added benefit that the sensor connecting leads can emerge from the endoscope umbilically rather than adding to the complexity of the control section. An inherent limitation of the system is that unlike fluoroscopy neither the gas shadow of the intestine nor the bony structures can be seen; the benefits of the real time and overall view obtained, however, easily compensate for this.

In conclusion we have found that electromagnetic imaging of colonoscopy is both practical and beneficial. During the examination it was easy to locate with accuracy any lesions encountered, to assess the large variety of loops that formed during intubation, and to visualise the manoeuvres necessary to straighten the colonoscope and advance the tip. Because of limited availability of the imaging equipment we have so far been unable to continue formal clinical evaluation. We suspect, however, that the system will be of even greater benefit to trainees or less experienced endoscopists. When commercially available, we predict that electronic imagers will have an important impact on training and performance of colonoscopy and should eventually become standard equipment in most endoscopy units.

The authors wish to thank Anne Morden, Rowen Pitt, and all the endoscopy staff of the Ipswich General Hospital.

This paper was presented at the March 1994 British Society of Gastroenterology Meeting and published in abstract form Gut 1994; 35 (suppl 2): S25.

1 Aldridge MC, Sim AJW. Colonoscopy findings in symptomatic patients without $x$-ray evidence of colonic neoplasia. Lancet 1986; ii: 833-4

2 Durdey P, Weston PMT, Williams NS. Colonoscopy or barium enema as initial investigation of colonic disease. Lancet 1987; ii: 549-51.

3 Lindsay DC, Freeman JG, Cobden I, Record CO. Should colonoscopy be the first investigation for colonic disease? BMF 1988; 296: 167-9.

4 Irvine EJ, O'Connor J, Frost RA, Shorvon P, Somers S, Stevenson GW, et al. Prospective comparison of doublecontrast barium enema plus flexible sigmoidoscopy colonoscopy in rectal bleeding: barium enema v colonoscopy in rectal bleeding. Gut 1988; 29: 1188-93.

colonoscopy in rectal bleeding. Gut 1988; 29: 1188-93.
Parry BR, Williams SM. Competency and the colonoscopist: a learning curve. Aust NZ F Surg 1991; 61: 419-22.

6 Saunders BP, Macrae FA, Williams CB. What makes colonoscopy difficult? [Abstract]. Gut 1993; 34 (suppl 1): T179.

7 Williams CB. Colonoscopy. In: Cotton PB, Williams CB, eds. Practical gastrointestinal endoscopy. 3rd ed. Oxford: Blackwell, 1990: 174-208

8 Bladen JS, Anderson AP, Bell GD, Rameh B, Evans B, Heatley DJT. Non-radiological technique for threedimensional imaging of endoscopes. Lancet 1993; 341: 719-22.

9 Williams CB, Guy C, Gilles D, Saunders BP. Electronic three-dimensional imaging of intestinal endoscopy. Lancet three-dimensional

10 Report by the Endoscopy Section Committee of the British Society of Gastroenterology. Future requirements for colonoscopy in Britain. Gut 1987; 28: 772-5.

11 Waye JD, Bashkoff MD. Total colonoscopy: is it always possible? Gastrointest Endosc 1991; 37: 152-4.

12 Ravi J, Brodmerkel GJ, Agarawal RM, Gregory DH, Ashok PS. Does prior abdominal or pelvic surgery affect length of insertion of the colonoscope? Endoscopy 1988; 20: 43.

13 Bat L, Williams CB. Usefulness of pediatric colonoscopes in adult colonoscopy. Gastrointest Endosc 1989; 35: 329-32.

14 Danesh BJZ, Spiliadis C, Williams CB, Zambartas CM. Angiodysplasia - an uncommon cause of colonic bleeding: colonoscopic evaluation of 1050 patients with rectal bleeding and anaemia. Int $\mathcal{f}$ Colorect Dis 1987; 2: 218-22. 